\section{Asthmatherapie bei Kindern: Auf die Compliance kommt es an!}

Asthmakranke Kinder, die inhalative Glukokortikoide brauchen und sie auch nehmen, sind besser dran als Kinder, die ein Glukokortikoidspray bräuchten, es aber nicht benutzen. Das haben die Ergebnisse einer Studie unterstrichen, in der die Compliance elektronisch kontrolliert wurde.

$\mathrm{n}$ eine Untersuchung zur Compliance bei asthmakranken Kindern haben brasilianische Pädiater 102 bis dato Glukokortikoid-naive Kinder im Alter zwischen fünf und 14 Jahren einbezogen, die an persistierendem Asthma litten. Die Patienten erhielten ein Rezept für Beclomethasondipropionat, von dem sie täglich 500-750 $\mu \mathrm{g}$ per Spray inhalieren sollten. Ein druckgesteuerter elektronischer Monitor, der an das Spray angeschlossen wurde, diente zur Überwachung der Therapie. Nachuntersuchungen gab es nach vier, acht und zwölf Monaten.

Die Forscher berechneten, wie sich die Therapietreue oder -untreue auf die Asthmabeschwerden auswirkte. Dabei galt das Asthma als kontrolliert oder unkontrolliert je nachdem, ob die Patienten tagsüber Beschwerden hatten oder in ihren Aktivitäten eingeschränkt waren, ob sie nachts
Symptome entwickelten, eine Akutmedikation benötigten oder Exazerbationen durchmachen mussten. Die Therapietreue wurde als prozentualer Anteil der Zahl

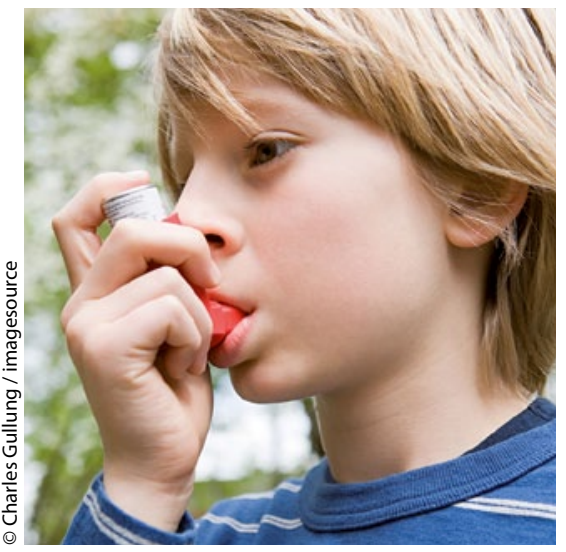

Treue zahlt sich auch in jungen Jahren aus - vor allem, wenn man Asthma hat. tatsächlich erfolgter Glukokokortikoidhübe zur Zahl der verordneten Hübe angegeben.

Wie kaum anders zu erwarten, waren Kinder mit guter Symptomkontrolle ihrer Behandlung in höherem Maße treu geblieben. Nach vier Monaten betrug die Treue in dieser Gruppe $60 \%$ und nach acht Monaten $56,2 \%$, nach zwölf Monaten allerdings nur noch $49,8 \%$. Kinder mit mangelnder Kontrolle ihrer Symptome wiesen Raten von $43,8 \%$ nach vier, $39,0 \%$ nach acht und 31,2\% nach zwölf Monaten auf. Im Effekt zeigte sich, dass eine Rate von $60 \%$ Therapietreue genügte, um das Asthma in Schach zu halten.

Fazit: Die brasilianischen Wissenschaftler raten, die Therapietreue von asthmakranken Kindern systematisch zu kontrollieren. Für einen Erfolg der Behandlung ist es dabei gar nicht nötig, den Idealwert von $100 \% \mathrm{zu}$ erreichen. Eine Treuerate von $60 \%$ genügt, um zumindest leichtere bis mäßige Formen der Erkrankung in den Griff zu bekommen.

Dr. Robert Bublak

Jentzsch NS et al. Adherence rate to beclomethasone dipropionate and the level of asthma control. Respiratory Medicine 2012; 106: 338-43

\section{Atopische Dermatitis: IL-31 reguliert Keratinozytendifferenzierung}

Von einer atopischen Dermatitis sind in Industrieländern 10-20\% der Kinder und $1-3 \%$ der Erwachsenen betroffen. Hautbiopsien der Patienten weisen eine erhöhte Expression des Interleukins IL-31 auf, die physiologische Relevanz von IL-31 ist jedoch bislang ungeklärt.

U $m$ der Frage auf den Grund zu gehen, welche Rolle IL-31 bei der atopischen Dermatitis (AD) spielt, nutzten Christian Cornelissen und Kollegen dreidimensionale organotypische Hautmodelle. Sie untersuchten so den Einfluss von IL-31 auf die Differenzierung von Keratinozyten.

Hautmodelle mit primären Keratinozyten und einem HaCaT-KeratinozytenZellmodell $(\mathrm{HaCaT}=$,human adult low calcium high temperature") mit induzierbarem IL-31-Rezeptor alpha (IL-31RA) zeigten, dass IL-31 negativ in den Differenzierungsprozess der Zellen eingreift.
Genexpressionsanalysen ergaben eine limitierte Anzahl von Genen, die abhängig von IL-31 dereguliert wurden, darunter auch Gene wie IL20 und IL24. Die Proliferation von HaCaT-Keratinozyten mit induzierbarem IL-31RA wurde durch Induktion eines Zellzyklusarrests nach IL31RA-Expression und IL-31-Stimulation gehemmt. Primäre Zellen und HaCaTZellen zeigten einen Differenzierungsdefekt, der durch eine reduzierte epidermale Dicke sowie eine insgesamt gestörte Ausbildung der Epidermis einschließlich Defekten in der basalen Zellschicht und des Stratum granulosum charakterisiert war.
Diese Differenzierungsdefekte waren assoziiert mit der Repression terminaler Differenzierungsmarker wie Filaggrin, ein für die Ausbildung der Hautbarriere essenzielles Protein, sowie der ceramidhaltigen Lipidschicht des Stratum corneum. Die pro-inflammatorischen Zytokine IL-20 und IL-24 waren teilweise verantwortlich für die Regulation der Filaggrin-Expression und somit für die Störung der terminalen Differenzierung.

Fazit: Diese Studie lässt vermuten, dass IL-31 ein wichtiger Regulator der Keratinozytendifferenzierung ist. Außerdem zeigt sie eine Verbindung zwischen der erhöhten IL-31-Expression in der Haut von $\mathrm{AD}$-Patienten und der Expression von Filaggrin. Dr. Prof. Dr. Jens M. Baron

Cornelissen C et al. IL-31 regulates differentiation and filaggrin expression in human organotypic skin models. J Allergy Clin Immunol 2012; 129: 426-33.e8 